binds Fc $\gamma$ Rs, as well as some nuclear proteins, including snRNPs. Pentameric (native) CRP has previously been suggested to inhibit production of IFNs in peripheral mononuclear cells (PBMCs) in response to ICs formed by autoantibodies against snRNP, an effect which was further investigated herein.

Methods PBMCs or magnetically (MACS) purified pDCs were retrieved from whole blood of healthy volunteers. Type I IFN gene transcription and production was stimulated by addition of snRNP containing ICs \pm pentameric CRP (pCRP) or monomeric CRP (mCRP) in different sequential order. IC formation was achieved through simultaneous addition of snRNP and bulk IgG, retrieved from an SLE patient with high levels of snRNP autoantibodies, directly to the cells. Type I IFNs and inflammatory cytokines were investigated using quantitative PCR, ELISA and cytometric bead array, and cells responsible for production of the IFNs were characterized using flow cytometry. For statistics a two-tailed t-test was performed.

Results pCRP had an inhibitory effect on the IFN gene expression in PBMCs after incubation with ICs, $p=0.044$ for IFN $\alpha 4$ and $p=0.047$ for IFN $\beta$ at the $4 \mathrm{~h}$ time-point compared to IC only. pCRP also showed a dose-dependent inhibitory effect on the type I IFN production in the cells. The monomeric form of CRP showed modest or no effect on IFN levels, $p=0.82$ for IFN $\alpha 4$ and $p=0.58$ for IFN $\beta$ at the $4 \mathrm{~h}$ time-point compared to IC only. A pre-incubation of the cells with pCRP increased the inhibitory effects compared to simultaneous addition of pCRP and ICs, suggesting that initial binding to the cells is a critical step for inhibition. Flow cytometry suggested that pDCs are the main producer of the type I IFNs. In addition, pCRP seems to have a more general inhibitory effect on type I IFNs, as seen in the reduction of IFN production in response to the TLR-9 ligand $\mathrm{CpG}$.

Conclusions pCRP has a distinct inhibitory effect on type I IFNs, which is largely not seen for the dissociated form of CRP (mCRP). The more general inhibitory effects shown by pCRP highlights its immune regulatory function in pathologies characterized by high production of type I IFNs. The identity of the initial receptors responsible for pCRP mediated effects, as well as of the involved signaling pathways, will be further investigated.

\section{P107 INTERFERON-INDUCED METABOLIC PERTURBATIONS SHAPE THE INFLAMMATORY STATUS OF HUMAN MONOCYTES: IMPLICATIONS FOR INNOVATIVE THERAPEUTIC ENGINEERING IN SLE AUTOIMMUNITY}

\footnotetext{
${ }^{1,2}$ Chrysoula Stathopoulou, ${ }^{3}$ Vasilis Ntassis, ${ }^{4}$ Aggelos Banos, ${ }^{2}$ Katerina Gkirtzimanaki, ${ }^{6}$ Antonis Myridakis, ${ }^{5}$ Christina Adamichou, $, 1,2,5$ Prodromos Sidiropoulos, $1,2,5$ George Bertsias. ${ }^{1}$ Rheumatology, Autoimmunity and Inflammation Laboratory, Medical School, University of Crete, Heraklion; ${ }^{2}$ Institute of Molecular Biology and Biotechnology, Foundation for Research and Technology Hellas, Heraklion; ${ }^{3}$ Dept. of Biology, University of Crete, Heraklion; ${ }^{4}$ Biomedical Research Foundation, Academy of Athens, Athens; ${ }^{5}$ Rheumatology Dept., University General Hospital, Heraklion, Greece; ${ }^{6}$ Dept. of Surgery and Cancer, Imperial College of London, St. Mary's Hospital, London, UK
}

10.1136/lupus-2020-eurolupus. 151

Immune cells have unique metabolic requirements to support the energetic and biosynthetic burden during their activation. Delineation of the metabolic tuning of immune cells could lead to novel strategies in treating metabolically- demanding processes including autoimmune diseases. Among innate effectors, monocytes have a distinct role in systemic lupus erythematosus (SLE) pathogenesis. We have previously described robust type-I interferon (IFN $\alpha$ ) signaling in patients with SLE. IFN $\alpha$-stimulated monocytes from healthy individuals (IFN-Mo) develop mitochondrial hyperpolarization and increased oxidative stress resembling SLE monocytes (SLE-Mo).

Here we sought to delineate the metabolic repercussion of IFN $\alpha$-mediated signaling that could explain metabolic shifts pertaining to autoimmunity. To this end, we combined transcriptomic data with metabolic flux analysis (Seahorse technology) and Gas Chromatography (GC-MS) in healthy monocytes, IFN-Mo and SLE-Mo. Our preliminary results indicate an increased, glucose-dose dependent glycolytic flux in IFN $\alpha$-treated healthy monocytes recapitulating the SLE-Mo phenotype. Blockade of hexokinase 2 (HK-2)-dependent glycolysis with the use of 2-DG inhibitor attenuated proinflammatory cytokine secretion and the expression of surface markers characteristic of activated monocytes, supporting the deregulated metabolic profile in SLE autoimmunity.

Combination of these data with targeted metabolomics (LCMS) analyses and the application of pathway-specific inhibitors are implemented in vitro to reverse the inflammatory state of SLE monocytes. Together, our data are expected to yield unique insights into the role of immunometabolism in SLE and the potential use of metabolites as novel therapeutic targets in autoimmunity.

\section{P108 OXIDATIVE STRESS IN NK CELL AND ITS CORRELATION WITH EXPRESSION OF KILLER IMMUNOGLOBIN RECEPTORS IN SLE PATIENTS}

Archana Bhatnagar, Ankit Tandon. Dept. of Biochemistry, Panjab University, Chandigarh, India

\subsection{6/lupus-2020-eurolupus. 152}

Background Oxidative stress i.e. accumulation of reactive oxidative species has been found to be implicated pathogenesis of many autoimmune diseases including systemic lupus erythematosus. Although our body has natural process of scavenging reactive oxidative species but whenever balance inclines towards accumulation, oxidative stress begins to build in. These accumulated ions lead to damage at cellular level and at molecular levels also. In our NK cell specific study we evaluated oxidative stress and expression level of killer immunoglobulin receptors. Killer cell immunoglobulin like receptors binds to mhc class 1 receptors. They work in antagonistic manner they are either activating for NK cell activity or inhibiting. So the balance between two categories is critical for self tolerance. We have evaluated expression level of kir2dl4 which binds to HLA-G ligand and activating in nature and KIR3DL1 on other hand interacts with HLA-Bw4 and prevent NK cell killing of healthy cells.

Methods Lupus Patient and healthy subjects Lupus patients are enrolled from outpatient department (OPD) of rheumatology clinic, PGIMER, Chandigarh.

Flowcytometric analysis $\mathrm{PBMC}$ isolated were incubated with antibodies conjugated to APC,PE, PerCP/cy5.5 for surface staining of antigens cd56, kir2dl4 and kir3dl1. DCFDA dye based analysis was al done for estimation cellular ROS levels. 
Quantitative real-time polymerase chain reaction: NK cells were sorted from using isolation kit from stemcell ${ }^{\mathrm{TM}}$ (cat no.17955). cDNA was synthesized from RNA separated from isolated nk cells. Gene primers were run on the StepOnePlus RealTime PCR Systems and analyzed with StepOne Software V2.1 (Applied Biosystems New York, USA).

Results Fraction of cd3-cd56 in pbmc (\%) were reduced in SLE patients $(5.395 \% \pm 0.6900 \mathrm{~N}=27)$ as compared to healthy control $(10.32 \pm 1.729 \mathrm{~N}=16)$, P Value0.0037

1. ROS increased in $\mathrm{cd} 3-\mathrm{cd} 56+$ cells of SLE patients as compared to healthy control.

2. Cd56 dim and cd56 bright cells of SLE have significantly higher fraction expressing kir2dl4.

3. Lower Cd56 dim cells of SLE patients express kir3dl1 on surface.

4. Mean mRNA level in SLE patients of HMOX-1 is 2.56 times higher as compared to healthy control. $(\mathrm{p}-\mathrm{value}=0.0532)$

5. Mean mRNA Expression level of kir3dl1 are not significantly different in SLE patients and healthy control

6. Mean mRNA expression level of kir2dl4 is 20 times higher in SLE patients as compared to healthy control(pvalue $=0.0260$ )

Conclusion In our study NK cell of patients have been found more oxidatively stressed as compared to healthy and Increased mRNA expression of HMOX-1 also indicate same. Significantly increased expression of KIR2dl4 at mRNA and surface expression may be responsible for killing HLA-G bearing self cells. Surface expression of Kir3dl1 has been found to be significantly decreased in CD 56 dim cells, probable consequence is reduced tolerance. Although we could find any significant correlation of mRNA expression kir2dl4 and kir3dl1 with that of hmox-1, so cannot conclude any relationship between these receptor and oxidative stress.

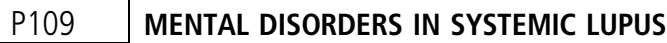 ERYTHEMATOSUS AND ANTIPHOSPHOLIPID SYNDROME PATIENTS}

${ }^{2}$ Anastasia Borisova, ${ }^{1}$ Fariza Cheldieva, ${ }^{1}$ Tatiana Lisitsyna, ${ }^{1}$ Tatiana Reshetnyak, ${ }^{2}$ Dmitry Veltishchev, ${ }^{2}$ Olga Seravina, ${ }^{2}$ Oksana Kovalevskaya. ${ }^{1}$ Research Institute of Rheumatology named after V.A. Nasonova, Moscow; ${ }^{2}$ Moscow Research Institute of Psychiatry, Serbsky NMRC PN MoH, Moscow, Russian Federation

\subsection{6/lupus-2020-eurolupus. 153}

Background Mental disorders (MD) in systemic lupus erythematosus (SLE) and antiphospholipid syndrome (APS) patients has been poorly described and recognized.

Objective To describe the frequency and spectrum of mental disorders in SLE and APS patients.

Methods 71 patients with mean $(\mathrm{M} \pm \mathrm{SD})$ age $38,8 \pm 11,7$ years were enrolled in the study: 21 of proven SLE patients, 26 SLE with secondary APS and 24 - with proven primary APS (PAPS). $54(76,1 \%)$ patients were women. SLE activity was measured by SLEDAI scale. MD were diagnosed by psychiatrist in accordance with the Hospital Anxiety and Depression Scale (HADS) and ICD-10 in semi-structured interview. The severity of depression and anxiety was evaluated by Montgomery-Asberg Depression Rating Scale (MADRS) and Hamilton Anxiety Rating Scale (HAM-A). CD were diagnosed with psychology and neuropsychology methods.
Abstract P109 Table 1 Prevalence of mental and cognitive disorders in SLE, SLE+APS and PAPS patients

\begin{tabular}{|c|c|c|c|c|}
\hline Sample characteristics & SLE, $n=21$ & $\begin{array}{l}\text { SLE+APS, } \\
n=26\end{array}$ & $\begin{array}{l}\text { PAPS, } \\
n=24\end{array}$ & $\mathbf{P}$ \\
\hline & 1 & 2 & 3 & \\
\hline \multirow[t]{3}{*}{ Gender (malelfemale), n (\%) } & 0121 & 6120 & $11 \backslash 13$ & Ns \\
\hline & $(0 \% \backslash 100 \%)$ & $(23,1 \%$ & $(45,8 \%$ & \\
\hline & & $176,9 \%)$ & $154,2 \%)$ & \\
\hline Age, years & $36,9 \pm 14,7$ & $39,3 \pm 10,6$ & $39,8 \pm 10,2$ & Ns \\
\hline SLEDAl, point & $6,4 \pm 5,7$ & $6,8 \pm 5,4$ & - & ns \\
\hline \multicolumn{5}{|l|}{ Mental disorders, n (\%) } \\
\hline Anxiety-depressive disorders: & $20(95,2 \%)$ & $18(69,2 \%)$ & $19(79,2 \%)$ & $P_{1-2}=0,03$ \\
\hline - recurrent depressive disorder & $10(47,6 \%)$ & $9(34,6 \%)$ & $10(41,7 \%)$ & ns ns ns ns \\
\hline - single depressive episode & $2(9,5 \%)$ & - & $1(4,2 \%)$ & \\
\hline - dysthymia & $6(28,6 \%)$ & $9(34,6 \%)$ & $6(25,0 \%)$ & \\
\hline - generalized anxiety disorder & $2(9,5 \%)$ & - & $2(8,3 \%)$ & \\
\hline Bipolar disorder & $1(4,8 \%)$ & $2(7,7 \%)$ & - & ns \\
\hline Schizotypal disorder & $2(9,5 \%)$ & $2(7,7 \%)$ & $1(4,2 \%)$ & ns \\
\hline Vascular dementia & - & $2(7,7 \%)$ & $1(4,2 \%)$ & ns \\
\hline Epileptic syndrome & $1(4,8 \%)$ & $6(23,1 \%)$ & $3(12,5 \%)$ & ns \\
\hline \multicolumn{5}{|l|}{ Cognitive impairment, n (\%) } \\
\hline $\begin{array}{l}\text { mild and moderate cognitive } \\
\text { impairment }\end{array}$ & $14(66,7 \%)$ & $18(69,2 \%)$ & $21(87,5 \%)$ & $P_{1-3}<0,05$ \\
\hline severe cognitive impairment & $4(19,0 \%)$ & $5(19,2 \%)$ & $2(8,3 \%)$ & ns \\
\hline
\end{tabular}

Results The patients with SLE, SLE+APS and PAPS didn't differ in age, but in PAPS group men met more often. The groups didn't differ in SLE activity. The rate of MD (mainly anxiety-depressive disorders) were high, highest in SLE patients. Recurrent depressive disorder met more often. The prevalence of anxiety-depressive disorders: recurrent depressive disorder, single depressive disorder, dysthymia, generalized anxiety disorder was significantly higher in SLE than in SLE+APS groups $(p=0,03)$. Bipolar disorder didn't meet in PAPS patients. Epileptic syndrome met more often (ns) in SLE+APS patients. The prevalence of schizotypal disorder was higher (ns) in patients with SLE (SLE and SLE+APS groups) (also higher than in population). Vascular dementia was diagnosed more often (ns) in APS patients (SLE+APS and PAPS groups). The rate of cognitive difficulties was very high in all groups, with predominance of mild and moderate impairment.

Conclusion Chronic depressive and cognitive disorders are typical for patients with SLE, SLE+APS and PAPS. Mild and moderate cognitive disorders diagnosed in PAPS-patients significantly more often $(p<0,05)$ than in SLE patients without APS.

\section{P110 LUPUS EUROPE - EXERCISE PROGRAM FOR LUPUS PATIENTS}

${ }^{1,2,3}$ Jeanette Andersen, ${ }^{1}$ Alain Cornet, ${ }^{1}$ Kirsi Myllys, ${ }^{1}$ Anne Charlet, ${ }^{1}$ Annemarie Sluijmers, ${ }^{1}$ Helga Ovens. 'Lupus Europe, Essex, UK; ${ }^{2}$ Lupus DK, Denmark; ${ }^{3}$ EULAR PARE Board, Zurich, Switzerland

\subsection{6/lupus-2020-eurolupus.154}

Background Up to $80 \%$ of all Lupus patients experience fatigue and most of them report this as the most severe symptom. One of the major causes of morbidity in SLE patients is 